\title{
Éditorial : La santé mentale en migrations internationales
}

Editorial: Mental Health in International Migration

Editorial: La salud mental en las migraciones internacionales

\section{Véronique Petit et Simeng Wang}

\section{(2) OpenEdition}

Édition électronique

URL : https://journals.openedition.org/remi/10489

DOI : $10.4000 /$ remi. 10489

ISSN : $1777-5418$

Traduction(s) :

Editorial: Mental Health in International Migration - URL : https://journals.openedition.org/remi/14302 [en]

Éditeur

Université de Poitiers

Édition imprimée

Date de publication : 1 octobre 2018

Pagination : 7-20

ISBN : 979-10-90426-62-7

ISSN : 0765-0752

Référence électronique

Véronique Petit et Simeng Wang, «Éditorial : La santé mentale en migrations internationales », Revue européenne des migrations internationales [En ligne], vol. 34 - n² et $3 \mid 2018$, mis en ligne le 28

décembre 2018, consulté le 15 avril 2022. URL : http://journals.openedition.org/remi/10489; DOI : https://doi.org/10.4000/remi.10489 


\section{Editorial}

\section{Véronique Petit ${ }^{1}$ et Simeng Wang ${ }^{2}$}

La Revue Européenne des Migrations Internationales n'a pas jusqu'alors consacré de dossier à la santé mentale en migrations. II nous a semblé nécessaire de combler cette lacune sans prétendre pour autant traiter de l'ensemble des questionnements qui auraient pu être inclus à l'intérieur de cette thématique riche. Plusieurs motivations expliquent cette volonté. La première s'inscrit dans le souhait de réagir face à certaines dérives politiques et sémantiques dans nombre de sociétés européennes caractérisées par un contexte sociopolitique marqué par la "crise des migrants". Un numéro spécial consacré à la santé mentale en migrations permet de tenir un discours savant reposant sur des connaissances empiriques qu'elles soient issues de la clinique ou des sciences sociales en réponse à certaines injonctions politiques et aux dérives populistes à l'encontre des "migrants malades" qui se diffusent de plus en plus largement dans I'espace médiatique et politique. En s'intéressant aux interactions entre migrations internationales et santé mentale il devient possible à partir d'observations précises de rappeler la réalité et la complexité des conditions sociales des migrations, d'examiner les pratiques de santé des migrants comme un indicateur de "l'accueil" leur étant réservé, des liens qu'ils entretiennent avec leur famille et de leur propre capacité d'agir durant leur quête thérapeutique. Derrière cette attention aux faits et à la prudence que les analyses requièrent, il s'agit de dénoncer des raccourcis porteurs de contre-vérités, d'autant plus dangereux qu'ils sont portés par des personnes investies de pouvoirs et de responsabilités. Citons à titre d'exemple, I'ancien ministre de I'Intérieur, Gérard Collomb, qui à la suite des attentats en Espagne en 2017 a déclaré vouloir mettre en place "une mobilisation de l'ensemble des hôpitaux psychiatriques et des psychiatres libéraux de manière à essayer de parer à cette menace terroriste individuelle", "ces protocoles" devraient être mis en place "lorsqu'un certain nombre de gens ont des délires autour de la radicalisation islamique ${ }^{3}$. Ces propos ont soulevé de vives critiques de la part du corps médical qui n'a pas manqué de les déconstruire et de les récuser. En effet, le discours de I'ancien

\footnotetext{
1 Démographe, Professeur, CEPED, Université Paris Descartes - IRD, 45 rue des Saints Pères 75006 Paris; veronique.petit@parisdescartes.fr

2 Sociologue, Chargée de recherche au CNRS, CERMES3 (CNRS-EHESS-INSERMUniversité Paris Descartes), 7 rue Guy Môquet, BP 8, 94801 Villejuif Cedex ; simeng.wang@cnrs.fr

3 Gourion David (2017) Terrorisme : «les psychiatres n'ont pas vocation à collaborer avec le ministère de l'intérieur ", Le Monde, 21/08/2017, [en ligne]. URL : https://www.lemonde. $\mathrm{fr} / \mathrm{idees} / \mathrm{article} / 2017 / 08 / 21 / \mathrm{les}-\mathrm{psychiatres-n-ont-pas-vocation-a-collaborer-avec-le-minis-}$ tere-de-I-interieur_5174728_3232.html
} 
ministre de l'Intérieur repose sur de fausses et dangereuses idées associant plus ou moins implicitement maladie mentale, religion et terrorisme. Insidieusement, ce discours en associant une religion et une maladie à la sécurité de l'État remet en question le droit des personnes issues de l'immigration à vivre légitimement leurs croyances et leurs maladies. II s'inscrit dans le prolongement du régime de suspicion généralisé développé à l'encontre des migrants et interroge de facto la place que nous réservons aux étrangers dans nos sociétés et notre capacité à entendre leurs voix et leurs multiples souffrances (voir la chronique d'actualité de Marie-Caroline Saglio-Yatzimirsky et Laure Wolmark).

\section{La santé mentale des migrants, quelles spécificités?}

Les études sur la santé mentale des migrants internationaux ne sont pas sans lien, d'une manière ou d'une autre, avec l'ethnopsychiatrie, née au cours du XXe siècle d'un mariage entre l'ethnologie et la psychiatrie. Pour Georges Devereux, père fondateur de l'ethnopsychiatre, elle "doit être une démarche “métaculturelle', c'est-à-dire qui ne se limite pas à l'ethnographie d'une société, mais qui transcende les différences grâce à une compréhension générale de la Culture, au sens d'une théorie générale de la culture... II existe un cadre théorique susceptible de rendre pertinente la distinction entre normal et pathologique, quelle que soit la culture" (Rechtman, 2018a : 93-94). Depuis, des recherches s'intéressent à l'introduction et la diffusion de la psychiatrie au sein des empires coloniaux en tant que discipline médicale et instrument de régulation sociale (Collignon et al., 1977; Collignon, 1999), à la confrontation entre ce savoir biomédical et les représentations locales de la maladie (Beneduce, 1997; Ortigues et Ortigues, 1966; Collomb, 1967), et à I'héritage complexe que constituent les institutions psychiatriques (Storper-Perez, 1974; Keller, 2007; Kilroy-Marac, 2009). Certaines recherches ont également cherché à restituer les conséquences des évolutions socioéconomiques et politiques (urbanisation, industrialisation, prolétarisation, paupérisation par exemple) sur les conditions de vie et comment elles pouvaient se répercuter sur la santé mentale des individus (Comaroff et Comaroff, 1987; Thomas et Znaniecki, 1998). Dans de nombreux travaux, on constate que le monde urbain est associé à la mobilité spatiale (migrations internes, exode rural, migrations internationales), à l'individuation et à l'accélération du temps. Les migrants doivent alors s'adapter à ce nouvel environnement social, qui est décrit comme pathogène en raison des nouveautés et des violences que les individus doivent gérer. Les décolonisations des années 1970 ont déplacé la question de l'altérité en psychiatrie et du rôle de la culture dans l'expression des maladies, en l'important des terrains exotiques (Fassin, 1999 et 2000a; Collignon, 1999 et 2006) vers le cœur des sociétés occidentales. À partir de cette date, l'ethnopsychiatrie qui jusqu'alors restait étroitement dépendante des territoires coloniaux, est devenue une pratique urbaine à l'égard des populations immigrées et réfugiées (Rechtman, 2018a : 97).

En effet, le psychisme se dévoile grâce à la médiation de la langue, de la gestuelle, des émotions et des attitudes. L'expression des souffrances psychiques varie considérablement d'une culture à l'autre. Lorsqu'il s'agit d'un sujet migrant, il peut s'avérer une plus grande altérité dans les relations thérapeutiques. C'est la raison pour laquelle l'ethnopsychiatrie, la psychiatrie transculturelle, la psychothérapie, la psychanalyse ont emprunté des connaissances 
anthropologiques pour appréhender le psychisme à travers les différences culturelles. Pourtant, être sensible à la variabilité "culturelle" n'égale pas la réduction aux explications culturalistes dans l'interprétation du psychisme. Sortant d'une dichotomie entre l'Occident et le reste du monde, les études contemporaines sur la santé mentale et les souffrances psychiques dans les contextes des migrations internationales sont invitées à prendre l'ensemble des sphères du monde contemporain. Puisque "d'une période historique à une autre, mais aussi d'un groupe social à un autre, on n'exprime pas son intimité avec les mêmes mots, on n'use pas des mêmes expressions, on n'utilise pas la même gestuelle" (Rechtman, 2018b : 402). Étudier la santé mentale en migrations internationales donne ainsi à voir comment un individu effectue le travail d' «ajustement du moi " (Ehrenberg, 2000) dans les contextes de mobilités spatiales et sociales; et comment les migrants se positionnent face aux normes sociales de désignation et d'assignation aux statuts de "malade" et de "fou" à la fois dans son pays d'origine et dans la société d'accueil (Rechtman, 2000).

\section{La fécondité du prisme de la santé mentale pour les études migratoires: les échelles analytiques micro, méso et macro}

\section{Subjectivité et vie intime des migrants}

La santé mentale et les souffrances psychiques constituent avant tout un prisme privilégié pour l'étude de la vie intime d'un individu. Grâce aux récits, aux discours et aux paroles, médiateurs du psychisme et révélateurs de la subjectivité (Ochs et Capps, 1996; Ochs et Sterponi, 2003), des chercheurs sont amenés à examiner comment les patients et leur entourage puisent dans les interprétations locales - représentations, rituels, imaginaires - afin de donner du sens à des parcours migratoires marqués par la maladie (Sylla et Mbaye, 1990-1991) et remettant en question le projet migratoire initialement construit. Travailler sur la santé mentale des personnes engagées dans une expérience migratoire, c'est ainsi prendre en considération la voix des migrants en souffrance et s'interroger les conditions sociales de leurs narrations, de leurs dits et non-dits. Comment endossent-ils leur rôle de "malade" ou de personnes désignées comme "folles"? Même si certains migrants sont soumis à des violences extrêmes au cours de leur voyage ou à des conditions de vie précaires, ils demeurent des acteurs de leur mobilité, et de leur trajectoire sociale, à laquelle participent l'expérience migratoire et les vécus de souffrance et de maladie. Entreprendre une quête thérapeutique, $c^{\prime}$ est aussi affirmer la prééminence de la vie, la reconquête d'une dignité perdue ou violée, s'interroger existentiellement sur le sens de sa trajectoire migratoire et des multiples expériences qui lui sont liés indissolublement. C'est devenir "un autre soi-même pour soi» (Ricœur, 1987), car la maladie en tant que fait individuel et social oblige à s'interroger et à mettre en perspective son histoire personnelle et sa propre trajectoire.

\section{Configurations familiales et relations intergénérationnelles}

Penser la santé mentale de migrants nécessite inévitablement de prendre en compte la configuration familiale du patient dans sa globalité, puisqu'un itinéraire de soin ne peut se détacher d'une trajectoire sociale qui lie le pays d'origine 
et le pays d'accueil (Sayad, 1999; Wang, 2017; Muel-Dreyfus, 2018; Pryor et al., 2019). Fréquemment investi de multiples attentes (financières, sociales, matérielles, affectives, symboliques, etc.) par sa famille et sa parenté, un migrant international peut se retrouver dans une logique de dette, dette économique, mais également morale (Wang, 2014). Le sens donné à la maladie et les stratégies de dissimulation face à la maladie, à la honte, à l'impact que les troubles psychiques peuvent avoir sur la réalisation du projet migratoire, modifient considérablement les dynamiques familiales et des liens entre le migrant et son entourage familial (Ly et al., 2014). Prenons l'exemple de familles chinoises à Paris où un enfant présente des difficultés psychologiques ou troubles mentaux, leur prise en charge et itinéraire thérapeutique sont conditionnés par les trajectoires migratoires de la famille tout entière, dans la prise de décision pour inscrire l'enfant à la MDPH (Wang, 2013b) comme dans la démarche de régularisation pour raison médicale (Wang, 2016b). À ce titre, le prisme de la santé mentale et des souffrances psychiques s'avère fécond puisqu'il révèle les relations familiales et intergénérationnelles dans lesquelles vivent le migrant, et qu'il nous renseigne également la transformation de ces relations familiales au cours de la trajectoire migratoire, d'avant l'émigration à l'après-immigration.

\section{Relations thérapeutiques et modes de prises en charge}

Étudier la santé mentale dans un contexte de migration internationale, permet à une autre échelle analytique de discuter des relations soignant/ soigné et des modes de prises en charge - sanitaire, sociale et juridique - des migrants. L'accès à la santé (mentale) des migrants constitue un double prisme : elle constitue d'une part un révélateur de l'offre de soin disponible, de sa qualité et de ses modalités d'accès; et d'autre part, elle donne une lecture des politiques migratoires et des conditions d'incorporation dans les pays d'immigration. Les travaux existants traitent des approches cliniques (Rechtman et Welsh, 1993); de la place de la culture dans le soin que ce soit dans les pays de départ ou d'arrivée des migrants (Nathan, 2000; Fassin, 1999 et 2000a; Wang, 2013b); des pratiques et enjeux de l'interprétariat et de la médiation (Wang, 2013a; Béal et Chambon, 2015; Blommaert, 2001; Belkacem, 2015; Wang, 2016c). Ces travaux ont pour commun d'interroger les politiques publiques au croisement de la migration et de la santé, l'organisation du système de soins, et plus largement l'accueil de migrants dans les pays d'installation, et les phénomènes de retour aux pays d'origine pour soins et traitement. Observer la santé mentale en migration implique de s'intéresser également aux interactions entre plusieurs types d'acteurs autour des prises en charge des migrants. Les personnes vivant avec un trouble psychique et une difficulté psychologique peuvent elles-mêmes engager une quête thérapeutique, ou se voir proposer une prise en charge spécifique étant située dans une configuration sociale spécifique : avec les statuts de "réfugié" ou de "mineur isolé ", être accueillie dans un camp, avoir commis une déviance dans l'espace public, vivre un retard scolaire, etc.

\section{Pluralisme médical et circulations en santé}

Enfin, s'intéresser sur la santé mentale en contexte migratoire, c'est aussi une manière d'étudier les rencontres entre différents systèmes médicaux et la circulation transnationale des savoirs et des pratiques de soins. Le parcours migratoire engendre des parcours thérapeutiques parfois complexes en élargissant la 
gamme des soins possibles dans des contextes de pluralisme thérapeutiques ici comme là-bas. Le migrant en tant qu'acteur de la mondialisation - soignés comme soignants - est un passeur par excellence de normes médicales et paramédicales, et de pratiques thérapeutiques, et de transferts immatériels en particulier dans les domaines de la formation des professionnels de santé. En effet, tout migrant ne se contente pas de se soigner en biomédecine, les recours aux soins dits "alternatifs" en santé mentale et les pratiques de soins à l'international sont observés de manière récurrente chez les migrants internationaux (Crenn et al., 2010; Wang, 2012). La mobilisation de ces ressources multiples et leurs usages doivent être interrogés au regard des processus de (re)socialisation, des processus de différenciation sociale, et des trajectoires et mobilités sociales.

\section{Une thématique jusqu'à présent peu abordée dans la REMI}

Alors que la question des liens entre santé mentale et expériences migratoires apparaît relativement tôt dans l'histoire des sciences sociales, notamment étudiée par l'École de Chicago (Faris et Dunham, 1939), elle demeure finalement peu traitée en tant qu'objet central dans la Revue Européenne des Migrations Internationales. Seul un article renvoie explicitement dans son titre à cette question (Devarenne-Megas, 2003). Même la question de santé, vraisemblablement moins pointue que celle de la santé mentale, reste relativement peu abordée. Entre 1980 et 2019, la REMI n'a publié qu'un seul dossier directement consacré aux "Expériences de la santé en migration " coordonné par Cognet, Hoyez et Poiret (2012). Les coordinateurs et les contributeurs mettaient alors I'accent sur l'analyse des inégalités et les discriminations dont sont victimes les migrants comparativement aux individus nés français en France, complétant ainsi la première synthèse de Berchet et Jusot (2012) et approfondissant la perspective initiée par Fassin (2000b). Dans ce numéro, sont examinés durant la trajectoire migratoire l'accès aux soins dans le cas de différentes pathologies (cancer, VIH, santé maternelle, soins palliatifs), les effets des conditions de vie (logement, emploi) sur la santé et les interactions entre l'accès aux soins et différents dispositifs administratifs.

Toujours dans la REMI, une vingtaine d'articles ont été consacrés à la question de la santé en migration à travers différentes thématiques : les parcours thérapeutiques, les pratiques de santé selon le statut administratif, le genre ou l'origine ethnique (Aumond, 2017; Alpes, 2017), les effets des discriminations, les inégalités entre migrants et personnes appartenant au groupe majoritaire, la place de la santé dans la protection accordée aux réfugiés (Gómez Martín, 2016), les mobilités des professionnels de la santé (Blain et al., 2014) et leur regard sur la situation migratoire ou coloniale (Moussy, 2015), la santé comme prisme de l'intégration, la contribution des migrants considérés comme "acteurs du développement" des systèmes de soins des pays de départ (Ndione et Lombard, 2004). À défaut d'un grand nombre d'articles, les occurrences "santé " et "santé mentale" reviennent plus fréquemment, à travers l'analyse d'interactions confrontant des profils de migrants à des contextes sociaux, politiques et admi- 
nistratifs spécifiques engendrant vulnérabilités et violences de multiple nature ${ }^{4}$. Ce qui traduit l'intrication des multiples dimensions de la santé dans les trajectoires et les expériences migratoires qui marquent les corps comme les esprits.

\section{Présentations des contributions à ce numéro}

Les articles qui composent ce dossier s'inscrivent dans différentes disciplines : anthropologie, sociologie, démographie, psychologie, médecine, géographie. Cette pluralité engendre des approches et des méthodologies spécifiques ainsi que des terrains d'enquêtes variés. La pluralité des profils de migrants et des situations décrites dans ce dossier permet d'aller au-delà de la question du syndrome du stress post-traumatique (SSPT), fortement associé à la santé mentale en migration, mais de montrer la diversité des souffrances psychiques, pouvant affecter chacun issu d'un parcours migratoire.

Dans ce dossier sur la santé mentale en migrations internationales, la santé psychique du migrant est saisie à différents moments de la trajectoire migratoire. Trois articles s'intéressent aux premiers temps qui suivent l'arrivée dans I'Union européenne en France et en Grèce, les exilés font alors le récit des tragédies qu'ils ont traversées, des épreuves qu'ils vivent (I'attente, la suspicion, le statut de survivant, l'angoisse du rapatriement) et des traumatismes physiques et psychiques qu'elles impriment durablement en eux. À travers le cas de réfugiés victimes de tortures arrivés en Grèce, Gail Womersley et Laure Kloetzer analysent la manière dont ceux-ci expriment leurs souffrances et leurs troubles mentaux. Elles s'intéressent en particulier à la manière dont est élaboré, construit et diagnostiqué le SSPT à travers les récits des migrants, les représentations et les pratiques des médiateurs culturels et des professionnels de santé autour du traumatisme. Elles rappellent l'importance fondamentale du médiateur culturel afin de saisir les singularités des expériences migratoires en effectuant une traduction entre des mondes, et non entre des langues, dans le contexte d'afflux de réfugiés dans I'Union européenne. La question de I'interprétariat traverse également I'article de Anaïk Pian, Anne-Cécile Hoyez et Simona Tersigni à propos de la prise en charge des migrants en santé mentale dans deux contextes spécifiques. L'analyse des dispositifs locaux (de Rennes et de Strasbourg) visant à prendre en charge les souffrances psychiques des migrants, les positionnements et les attentes des acteurs (professionnels de santé, associations, interprètes, migrants), la construction d'une relation triangulaire migrant-interprète-soignant révèlent l'imbrication d'enjeux thérapeutiques et politiques aux échelles locales. Les approches différenciées de l'interprétariat posent les questions de la professionnalisation de l'interprétariat - sa place, sa "juste" distance vis-à-vis des migrants comme des acteurs de santé - et des suivis thérapeutiques et sociaux des migrants. Quelle place ces acteurs réservent-ils aux explications culturelles dans la relation thérapeutique? Enfin, le texte de Lou Einhorn, Maud Rivière, Marielle Chappuis, Marie Chevelle et Sophie Laurence décrit la réponse en santé mentale et en soutien psychologique mise en place par cette une ONG dans ce qu'ils décrivent comme un contexte de "crise aux exilés". Outre le cadre théorique du dispositif d'aide thérapeutique

4 Citons à titre d'exemples les dossiers les plus récents illustrant cette perspective : 2017, $33(2) ; 2017,33(4) ; 2016,32(2) ; 2014,30$ (1). 
proposé, cet article revient sur les effets tragiques des conditions d' "accueil» réservé aux migrants dans le Calais. La violence dont ils sont les victimes n'est ni celle qui les a contraints à fuir ni celle de ceux qui les ont exploités au cours de leur parcours, mais la violence structurelle le pays d'arrivée. II s'agit pour les soignants de rétablir la possibilité de construction de relations de confiance dans un environnement peu propice au suivi thérapeutique, car ces bidonvilles-camps sont marqués par le stress, la précarité, le dénuement et l'incertitude quant aux lendemains.

D'autres articles s'attachent à resituer la santé mentale chez des migrants installés depuis des années dans le pays d'arrivée en s'interrogeant sur la question du lien qu'entretiennent les migrants avec leur famille au pays de départ. Si des recherches sur les transferts ont montré de quelle manière la participation des migrants à l'économie de leur famille au pays a des répercussions directes sur le bien-être de celle-ci, il est plus rare de disposer d'études montrant que les migrants en exil s'appuient aussi sur des ressorts affectifs et psychologiques dans les combats qu'ils mènent quotidiennement afin d'accéder à une régularisation de leur situation, d'obtenir un emploi ou un logement, et de voir leurs conditions s'améliorer. Julie Pannetier pour le groupe Parcours démontre à partir d'une enquête démographique l'effet positif et statistiquement significatif du soutien familial. Ce résultat - même s'il peut sembler être une évidence ou une banalité pour ceux qui disposent d'une expérience clinique - est corroboré grâce à une approche quantitative démographique.

Les articles s'intéressant aux contextes de départs des migrants ou aux pays d'origine pour les descendants de migrants permettent d'opérer un décentrement sur l'histoire et les conditions d'exercice de la psychiatrie dans des espaces postcoloniaux et dans des cultures marquées par un fort pluralisme thérapeutique (Melchior et al., 2012; Wang, 2016a). Là-bas comme ici, il s'agit de dessiner comment est structurée l'offre de soin à de multiples échelles (locale, régionale, nationale, transnationale), à travers quels types de prise en charge elle répond (ou non) aux souffrances des migrants et leurs descendants, en quoi elle s'articule ou non à une politique migratoire ou une politique de santé. L'accès aux soins permet d'appréhender les conditions d'existence des migrants et des ressources dont ils disposent, y compris pour exprimer la souffrance à être qui les habite et qui parfois se transmet tel un héritage indicible. Ces différentes dimensions permettent de dresser le contexte de production de leurs discours et de le réintégrer dans une histoire en mouvement. Plusieurs contributions à ce numéro mettent en avant les dynamiques et mouvements reliant le pays d'origine et celui d'arrivée. Dans une perspective psychologique et épidémiologique, Kossigan Kokou-Kpolou, Daniel Mbassa Menick, Charlemagne Simplice Moukouta et Élodie Gaëlle Ngameni ont appréhendé les effets du deuil d'un être cher chez des migrants ouest-africains en Europe selon leur statut et selon les possibilités d'effectuer un retour afin d'y effectuer certains rituels. François Sicot et Slimane Touhami, quant à eux, suivent les pas des migrants maghrébins et de leurs descendants au fil de leurs parcours thérapeutiques de part et d'autre de la méditerranée : comment vivent-ils la maladie mentale dans un contexte migratoire? Quelles réponses apportent-ils aux souffrances psychiques en fonction de leurs savoirs médicaux et paramédicaux? Auprès de quels acteurs vont-ils trouver un recours médical et/ou religieux? Enfin, le temps du retour des migrants dans leur pays est abordé à propos des migrants sénégalais dans 
I'article de Véronique Petit. Celui-ci prolonge la perspective soulevée précédemment en questionnant la solidarité familiale lorsque la maladie met à mal le projet migratoire, projet qui est l'objet d'un investissement collectif sans cesse réactivé. L'article montre que quelques soient les causes du retour, l'expérience migratoire modifie la position sociale du migrant et bouleverse son rapport aux autres, puisque le statut des migrants reste lié à leur réussite et à leur capacité de redistribution. Dans les contributions de Julie Pannetier et Véronique Petit, la perspective classique est renversée, car il ne s'agit plus de montrer de quelle manière les migrants soutiennent leurs parents au pays à travers les transferts sociaux ou financiers, mais de comprendre comment eux-mêmes sont profondément affectés par ces relations affectives transnationales et les dynamiques familiales dans lesquelles ils s'insèrent. Le migrant est aussi dépendant de sa famille s'il fallait rappeler cette forme d'économie morale.

\section{Décentrer le regard : pratiques transnationales de santé, immigrations non postcoloniales et recours aux soins dans le secteur privé}

Les articles composant ce dossier sont principalement centrés sur les primoarrivants, sauf I'article de François Sicot et Slimane Touhami, qui met en lumière des pratiques transnationales de santé mentale chez les descendants de migrants d'origine maghrébine. Ils montrent comment les parcours thérapeutiques et les représentations de la maladie sont marqués par la transmission de savoirs et de pratiques qui viennent compléter l'offre biomédicale en France. Ces processus de transmission croisent ceux de la mobilité sociale interrogent la proximité et les mécanismes d'appropriation de la psychiatrie, de la psychologie et de l'ensemble des savoirs médicaux et paramédicaux (psychothérapie, psychanalyse, médecines dites "alternatives", thérapies religieuses, etc.). Aux migrations des différentes catégories de thérapeutes, aux circulations des personnes malades se superposent également des circulations de produits de santé, de pratiques thérapeutiques, et de savoirs médicaux et paramédicaux. La diffusion de la psychiatrie comme celle d'ailleurs du concept de "santé mentale" devrait être davantage historicisée afin de s'ouvrir aux situations des pays qui, soit n'entrent pas dans une situation postcoloniale, soit ne disposent pas d'un système de soins psychiatrique hérité de la colonisation. On le voit dans le cas de la France, toutes les composantes de l'empire colonial n'ont pas bénéficié d'un investissement égal dans l'implantation de structures psychiatriques (Collignon, 2006). Et parmi les populations immigrées en France dépourvues de dimension postcoloniale, comme dans le cas de l'immigration chinoise, des facteurs sociologiques identifiés - par-delà des spécificités liées à chaque contexte d'émigrationimmigration historique, ethnique et national - , ont apporté plus largement des appréhensions sociologiques sur des questions transversales aux études des migrations : relations intergénérationnelles, mobilités sociales, relations thérapeutiques à l'épreuve de l'interculturel, circulations transnationales en santé, ou encore, accueil des migrants en France (Wang, 2018).

Même si les recours aux soins dans le secteur privé ne sont guère abordés dans ce dossier, il importe de déconstruire certaines images figées attribuées aux migrants : "pauvres", "peu ou non qualifiés", "usagers stratèges des soins du secteur public", afin de travailler plus finement sur la différenciation 
sociale dans l'analyse des recours aux soins des migrants. Parmi les recherches en sciences sociales françaises, la santé mentale de certaines catégories de migrants internationaux et leurs recours aux soins sont davantage étudiés : demandeurs d'asile et réfugiés (d'Halluin, 2009; Pestre, 2010; Halima et al., 2012; Saglio-Yatzimirsky, 2015 et 2018), migrants en situation irrégulière (Wang, 2016b) ou encore ceux vivant en grande précarité (Chambon et al., 2013; Roze et al., 2016). Alors que les souffrances psychiques de migrants qualifiés (Wang, 2015 et 2017), plus largement de ceux issus de classes moyennes et supérieures et leurs recours aux soins en santé mentale sont rarement abordées, notamment quand ils se soignent dans le secteur privé de la psychiatrie. II nous semblerait ainsi heuristique d'approfondir nos connaissances sur les liens entre la diversité de catégories de migrants internationaux et leur itinéraire thérapeutique en santé mentale; ainsi que sur la différenciation sociale de recours aux soins parmi des migrants internationaux, à travers la diversité des rapports sociaux de race, de sexe, de genre, de classe, d'origine ethnique, d'origine régionale, d'âge et de génération.

Enfin, comme perspectives de recherche, indépendamment des recherches sur les migrations internationales, la santé mentale connaît également un regain d'intérêt au niveau international pour deux raisons essentielles. Tout d'abord dans la perspective de la transition épidémiologique en cours, l'attention se porte de plus en plus sur les maladies non transmissibles et les maladies chroniques qui demandent un suivi à moyen ou long terme. Les recherches épidémiologiques mettent en exergue le décalage qui existe entre la charge morbide des troubles psychiques de mieux en mieux mesurée (Viggo et al., 2016; Charlson et al., 2014; Patel et al., 2018) et les ressources consacrées à leur prise en charge. Ce constat est dramatique en ce qui concerne les pays en développement et tout particulièrement l'Afrique, I'Organisation mondiale de la santé (OMS) estime que les pays en développement consacrent moins de $1 \%$ de leur budget de santé à la santé mentale (Addo et al., 2018; Bloom et al., 2011), qu'entre 75 et $85 \%$ des individus atteints de troubles mentaux graves n'y reçoivent aucun soin et rares sont les pays qui disposent de programmes ou de politiques de santé mentale. Le champ de la santé mentale au niveau international évolue également du fait de la question environnementale et de ses effets sur la migration. En effet, les projections démographiques liées aux déplacements et aux migrations environnementales en dépit de leur grande variabilité quant à l'estimation chiffrée de la catégorie des "réfugiés environnementaux» et à la définition de celle-ci laissent augurer de l'importance croissance de cette problématique dans les études migratoires à venir. Selon le Haut-Commissariat aux réfugiés des Nations unies, 250 millions de personnes d'ici à 2050 seront obligées de se déplacer dans le monde; d'autres méthodologies et estimations conduisent à des chiffres encore plus élevés. L'ensemble de ces contextes environnementaux, économiques, politiques, sanitaires et juridiques font conjointement émerger des mobilités internationales qui s'imposent parfois aux individus et façonnent leurs psychismes et pratiques thérapeutiques. La situation psychique des migrants déplacés, rapatriés, retournés, ou en situation de transit précaire, dans les pays de départ comme dans ceux d'arrivée, mérite ainsi d'être plus explorée par la recherche à l'ère de globalisation. 


\section{Références bibliographiques}

Addo Rebecca, Agyemang Samuel A., Tozan Yesim and Nonvignon Justice (2018) Economic burden of caregiving for persons with severe mental illness in sub-Saharan Africa: A systematic review, PLOS ONE, 13 (8), [online]. URL: https://www. ncbi.nlm.nih.gov/pmc/articles/PMC6084810/

Alpes Maybritt Jill (2017) Les dynamiques sociologiques entre contrôle des migrations et protection sociale : femmes migrantes sans papiers en quête d'hébergement à Paris, Revue Européenne des Migrations Internationales, 33 (4), pp. 135-157.

Aumond Florian (2017) Quand I'exceptionnel dure et le provisoire se normalise : les Centres d'Accueil et d'Orientation pour mineurs non accompagnés (CAOMI), Revue Européenne des Migrations Internationales, 33 (1), pp. 157-163.

Béal Arnaud et Chambon Nicolas (2015) Le recours à l'interprète en santé mentale : enjeux et problèmes, Rhizome, 55 (1), pp. 9-19.

Belkacem Lila (2015) Quand la clinique fait l'ethnique? Logiques performatives dans la médiation interculturelle pour familles migrantes, Genèses, 98, pp. 47-68.

Beneduce Roberto (1997) Aliénation mentale et altérité du sujet colonial : de quelques difficultés de l'émergence d'une relation thérapeutique basée sur le dialogue en Afrique, in Roberto Beneduce, Saperi, linguaggi e tecniche nei sistemi di cura tradizionali, Torino, L'Harmattan Italia, pp. 71-88.

Berchet Caroline et Jusot Florence (2012) État de santé et recours aux soins des immigrés : une synthèse des travaux français, Questions d'économie de la santé, 172 , pp. $1-8$.

Blain Marie-Jeanne, Fortin Sylvie et Alvarez Fernando (2014) Être médecin et immigrant au Québec : une identité professionnelle malmenée, Revue Européenne des Migrations Internationales, 30 (3), pp. 139-162.

Blommaert Jan (2001) Context is/as Critique, Critique of anthropology, 21, pp. 13-32.

Bloom D. E., Cafiero E. T., Jané-Llopis E., Abrahams-Gessel S., Bloom L. R., Fathima S., Feigl A. B., Gaziano T., Mowafi M., Pandya A., Prettner K., Rosenberg L., Seligman B., Stein A. Z. and Weinstein C. (2011) The Global Economic Burden of Noncommunicable Diseases, Geneva, World Economic Forum, 48 p.

Chambon Nicolas, Cochet Pierre et Le Goff Gwen (2013) Soigner des migrants précaires en psychiatrie publique, Écarts d'identité, pp. 38-44, [enligne]. URL : https://halshs.archives-ouvertes.fr/halshs-00978254

Charlson Fiona J., Diminic Sandra, Lund Crick, Degenhardt Louisa and Whiteford Harvey A. (2014) Mental and Substance Use Disorders in Sub-Saharan Africa: Predictions of Epidemiological Changes and Mental Health Workforce Requirements for the Next 40 Years, PLOS ONE, 9 (10), [online]. URL: https:// journals.plos.org/plosone/article?id=10.1371/journal.pone.0110208

Cognet Marguerite, Hoyez Anne-Cécile et Poiret Christian (Coord.) (2012) Expériences de la santé en migration, Revue Européenne des Migrations Internationales, $28(2)$, pp. 7-170.

Collignon René (2006) La psychiatrie coloniale française en Algérie et au Sénégal : esquisse d'une historisation comparative, Revue Tiers-Monde, 187, pp. 527-546. 
Collignon René (1999) Le traitement de la folie au Sénégal à l'époque coloniale, in Bernault Florence, Enfermement, prisons et châtiments en Afrique du $19 e$ siècle à nos jours, Paris, Khartala, pp. 227-258.

Collignon René, Osouf Patrick et Sylla Omar (1977)Thiaroye ou les avatars d'une institution, Psychopathologie africaine, 13 (1), pp. 81-111.

Collomb Henri (1967) Rencontre de deux systèmes de soins. À propos de thérapeutiques des maladies mentales en Afrique orientale, Social Science \& Medicine, 7 (8), pp. 623-633.

Comaroff Jean and Comaroff John (1987) The madman and the migrant: work and labor in the historical consciousness of a South African people, American Ethnologist, 14 (2), pp. 191-209.

Crenn Chantal, Hassoun Jean-Pierre et Medina F. Xavier (2010) Introduction : repenser et réimaginer I'acte alimentaire en situations de migration, Anthropology of food, 7, [en ligne] consulté le 25/02/2019. URL : https://journals.openedition. org/aof/6672

Devarenne-Megas Hanna (2003) Psychopathologie et insertion sociale des migrants polonais en France, Revue Européenne des Migrations Internationales, 19 (1), pp. 101-124.

Ehrenberg Alain (2000) La fatigue d'être soi. Dépression et société, Paris, Odile Jacob, $409 \mathrm{p}$.

Faris Robert E. Lee and Dunham H. Warren (1939) Mental Disorders in Urban Areas: an Ecological Study of Schizophrenia and other Psychoses, Chicago, University of Chicago Press.

Fassin Didier (2000a) Les politiques de l'ethnopsychiatrie. La psyché africaine, des colonies africaines aux banlieues parisiennes, L'Homme, 153, pp. 231-250.

Fassin Didier (2000b) Repenser les enjeux de santé autour de l'immigration, Hommes et Migrations, 1225, pp. 5-12.

Fassin Didier (1999) L'ethnopsychiatrie et ses réseaux : I'influence qui grandit, Genèses, 35, pp. 146-171.

Gómez Martín Carmen (2016) La génération sahraouie de la guerre : des études à Cuba à la migration économique en Espagne, Revue Européenne des Migrations Internationales, 32 (2), pp. 77-94.

Halima Zeroug-Vial, Le Goff Gwen, Benkorichi Malorie et Chambon Nicolas (2012) Etat des lieux national de la santé mentale des demandeurs d'asile et réfugiés au sein du dispositif national d'accueil. Mise en perspective problématique, Réseau Samdarra. Récits et paroles de migrants en quête d'asile. Quels enjeux? Quels effets sur la santé mentale?, Lyon, pp. 3-5.

Halluin Estelle ( $\left.d^{\prime}\right)$ (2009) La santé mentale des demandeurs d'asile, Hommes et migrations, 1282, pp. 66-75.

Keller Richard C. (2007) Colonial Madness: Psychiatry in French North Africa. Chicago and London, The University of Chicago Press, 320 p.

Kilroy-Marac Kathleen (2009) The impossible inheritance: Memory and Postocolonial subjectivity at the Fann Hospital in Dakar, Senegal, Columbia University, NY, Thesis for the degree of Doctor of Philosophy in Graduate School of Arts and Sciences. 
Laliberté Danièle (2007) Crises humanitaires, santé des réfugiés et des déplacés: un cadre analytique, Revue Européenne des Migrations Internationales, 23 (3), pp. 85-96.

Ly Mouhamed, Petit Véronique et Pizzolato Giulia (2014) La migration internationale face à la santé mentale au Sénégal : récits, discours et imaginaires, in Cécile Canut et Catherine Mazauric Éds., Les mots de la migration. Mise en récits et en images des migrations transafricaines, Paris, Éditions Le cavalier bleu, pp. 221-239.

Melchior Maria, Chastang Jean-François, Falissard Bruno, Galéra Cédric, Tremblay Richard E., Côté Sylvana and Boivin Michel (2012) Food Insecurity and Children's Mental Health: A Prospective Birth Cohort Study, PLoS ONE, 7 (12), [online]. URL: https://journals.plos.org/plosone/article?id=10.1371/journal.pone.0052615

Moussy Hugues (2015) Le regard des médecins topographes sur l'Algérie coloniale, Revue Européenne des Migrations Internationales, 31 (3), pp. 231-251.

Muel-Dreyfus Francine (2018) Abdelmalek Sayad : sociologie historique et sociologie clinique de l'émigration/immigration, in Isabelle Coutant et Simeng Wang Dir., Santé mentale et souffrance psychique. Un objet pour les sciences sociales, Paris, CNRS Éditions, pp. 115-117.

Nathan Tobie (2000) Psychothérapie et politique. Les enjeux théoriques, institutionnels et politiques de l'ethnopsychiatrie, Genèses, 38 (1), pp. 136-159.

Ndione Babacar et Lombard Jérôme (2004) Diagnostic des projets de réinsertion économique des migrants de retour : étude de cas au Mali (Bamako, Kayes), Revue Européenne des Migrations Internationales, 20 (1), pp. 7-27.

Ochs Elinor and Capps Lisa (1996) Narrating the self, Annual Review of Anthropology, 25, pp. 19-43.

Ochs Elinor and Sterponi Laura (2003) Analisi delle narrazioni, in Giuseppe Mantovani e Anna Spagnolli Eds., Metodi qualitativi in psicologia, II Mulino, pp. 131-158.

Ortigues Marie-Claude et Ortigues Edmond (1966) Edipe africain, Paris, Plon, $321 \mathrm{p}$.

Patel Vikram, Shekhar Saxena S., Lund Crick, Thornicroft Graham, Baingana Florence and Bolton Paul (2018) The Lancet Commission on global mental health and sustainable development, The Lancet, $46 \mathrm{p}$.

Pestre Élise (2010) La vie psychique des réfugiés, Paris, Payot et Rivages, 318 p.

Pryor Laura, Strandberg-Larsen Katrine, Nybo Andersen Anne-Marie, Rod Naja Hulvej and Melchior Maria (2019) Trajectories of family poverty and children's mental health: Results from the Danish National Birth Cohort, Social Science \& Medicine, 220, pp. 371-378.

Rechtman Richard (2018a) Aux origines de I'ethnopsychiatrie, in Isabelle Coutant et Simeng Wang Dir., Santé mentale et souffrance psychique. Un objet pour les sciences sociales, Paris, CNRS Éditions, pp. 73-97.

Rechtman Richard (2018b) Postface, in Isabelle Coutant et Simeng Wang Dir., Santé mentale et souffrance psychique. Un objet pour les sciences sociales, Paris, CNRS Éditions, pp. 401-405.

Rechtman Richard (2000) De la psychiatrie des migrants au culturalisme des ethnopsychiatres, Hommes et Migrations, 1225, pp. 46-61. 
Rechtman Richard et Welsh Geneviève (1993) Approche transculturelle des patients non francophones originaires du Sud-est asiatique dans le dispositif psychiatrique du XIIle arrondissement de Paris, Santé mentale au Québec, 18 (1), pp. 143-161.

Ricœur Paul (1987) Soi-même comme un autre, Paris, Édition du Seuil, 448 p.

Roze Mathilde, Vandentorren Stéphanie, Vuillermoz Cécile, Chauvin Pierre and Melchior Maria (2016) Emotional and behavioral difficulties in children growing up homeless in Paris. Results of the ENFAMS survey, European Psychiatry, 38, pp. 51-60.

Saglio-Yatzimirsky Marie-Caroline (2018) La voix de ceux qui crient. Rencontre avec des demandeurs d'asile, Paris, Alban Michel, 318 p.

Saglio-Yatzimirsky Marie-Caroline (2015) Kalapani : le trauma de la traversée dans la migration des demandeurs d'asile tamouls du Sri Lanka, Migrations Société, 161.

Sayad Abdelmalek (1999) La double absence. Des illusions de l'émigré aux souffrances de l'immigré, Paris, Le Seuil, $437 \mathrm{p}$.

Storper-Perez Danielle (1974) La folie colonisée, Paris, François Maspéro, 156 p.

Streiff-Fénart Jocelyne et Poutignat Philippe (2014) Vivre sur, vivre de la frontière : I'après transit en Mauritanie et au Mali, Revue Européenne des Migrations Internationales, 30 (2), pp. 91-111.

Sylla Omar et Mbaye Mor (1990-1991) Psychopathologie et migration : un cas de "wootal ", Psychopathologie africaine, 23 (3), pp. 353-363.

Thomas William I. et Znaniecki Florian (1998 [1919]) Le paysan polonais en Europe et en Amérique : récit de vie d'un migrant, Paris, Nathan, 446 p.

Viggo Daniel, Thornicroft Graham and Atun Rifat (2016) Estimating the true global burden of mental illness, The Lancet Psychiatry, 3, pp. 171-178.

Wang Simeng (2018)Étudier les souffrances des migrants : à partir du cas des Chinois en France, in Isabelle Coutant et Simeng Wang Dir., Santé mentale et souffrance psychique. Un objet pour les sciences sociales, CNRS Éditions, pp. 325-344.

Wang Simeng (2017) Illusions et souffrances. Les migrants chinois à Paris. Paris, Éditions rue d'Ulm, 220 p.

Wang Simeng (2016a) Souffrances psychiques et mobilité sociale ascendante : le cas des adolescents français d'origine chinoise à Paris, Hommes \& Migrations, 1314, pp. 11-18.

Wang Simeng (2016b) Entre stratégie et moralité. Des migrants chinois en situation irrégulière face à la " clause de maladie ", Politix, 114, pp. 205-227.

Wang Simeng (2016c) "Aidez-nous à comprendre vos Chinois! " Conditions de possibilités de la légitimation du sociologue en milieu psychiatrique, Genèses, 105, pp. 141-156.

Wang Simeng (2015) Des " soucis matrimoniaux " dans le contexte transnational : le cas des migrant·e.s chinois·es qualifié·e·s à Paris, Migrations Société, 27 (157), pp. 149-166. 
Wang Simeng (2014) «Enfant abandonné en Chine puis domestique en France. Que suis-je pour eux?! "Obligations familiales à rebours des enfants migrants d'origine chinoise à Paris, Enfances, Familles, Générations, 20, pp. 21-44.

Wang Simeng (2013a)Analyses sociologiques des pratiques de l'interprétariatmédiation en pédopsychiatrie : I'exemple des familles d'origine chinoise à Paris, Enfances \& Psy, 56, pp. 136-146.

Wang Simeng (2013b) " Handicapé ? C'est insulter ma descendance et aussi mes ancêtres! ". Négociations autour de l'inscription de l'enfant d'origine chinoise à la MDPH, Terrains \& travaux, 23, pp. 77-92.

Wang Simeng (2012) "Une vie qui est ailleurs " : une sociologie des troubles mentaux à travers le parcours d'un adolescent d'origine chinoise à Paris, Lien social et politiques, 67, pp. 233-248. 